\title{
Akademievorträge
}

Vortragsabend der Akademie der Wissenschaften zu Göttingen in der Vertretung des Landes Niedersachsen beim Bund in Berlin

15. Oktober 2013

\section{Bertram Brenig Genomische Aberrationen in caninen Mammakarzinomen und deren Nachweis in zellfreier plasmatischer DNA}

Bertram Brenig ${ }^{1}$, Julia Beck ${ }^{2}$, Silvia Hennecke ${ }^{1}$, Kirsten Bornemann-Kolatzki², Howard B. Urnovitz ${ }^{2}$, Stephan Neumann ${ }^{1}$, Philipp Ströbel ${ }^{3}$, Franz-Josef Kaup ${ }^{4}$, Ekkehard Schütz ${ }^{1,2}$

${ }^{1}$ Tierärztliches Institut, Georg-August Universität Göttingen

${ }^{2}$ Chronix Biomedical GmbH, Göttingen

${ }^{3}$ Abteilung Pathologie, Universitätsmedizin Göttingen UMG

${ }^{4}$ Deutsches Primatenzentrum, Göttingen

\section{Zusammenfassung}

Tumoren des Gesäuges sind die häufigste Krebsart bei Hündinnen, die eine große histopathologische Variabilität aufweisen. Jedoch ist über die genaue genomische Zusammensetzung dieser Tumore nur wenig bekannt. Im Rahmen einer Studie haben wir Mammakarzinome von fünf Hündinnen mit modernen Hochdurchsatzsequenzierungstechnologien auf strukturelle und Kopienzahlveränderungen im Vergleich zum somatischen Genom analysiert und diese mit einer „droplet“ digitalen PCR (ddPCR) validiert. Neben chromosomalen Aneuploidien konnten außerdem kleinere Deletionen und interchromosomale Fusionen in den Tumoren nachgewiesen werden. Häufig waren von den Aberrationen bekannte Oncogene, wie $c M Y C$ und KIT, betroffen. In vier der Tumoren konnte eine Deletion des proximalen Endes von Chromosom 27 nachgewiesen werden, in der das Tumorsuppressorgen Prefoldin 5 (PFDN5) gelegen ist. Mittels ddPCR konnte diese Deletion in 50\% der Mammakarzinome ( $N=20)$ detektiert werden. Zum Nachweis der Deletion in zellfreier plasmatischer DNA wurde eine Bruchpunkt spezifische PCR entwickelt. Bei einer Hündin konnten spezifische Signale noch ein Jahr nach der Operation festgestellt werden, welche auf eine Lungenmetastase zurückzuführen waren. Durch Sequenzierung der zellfreien plasmatischen DNA konnte gezeigt werden, dass sämtliche chromosomalen Aberrationen auch im Blut der Hündinnen nachweisbar waren. Neben spezifischen caninen zeigten die identifizierten Veränderungen Ähnlichkeiten mit humanen Tumoren. Unsere Ergebnisse zeigen, 
dass es moderne Hochdurchsatzsequenzierungstechnologien erlauben individuelle Tumormarker zu identifizieren, die eine gezielte Überwachung der Tumorentwicklung und Metastasierung ermöglichen.

\section{Einleitung}

Die häufigsten neoplastischen Erkrankungen bei Hündinnen sind Tumoren des Gesäuges, die mehr als 40\% aller Tumoren ausmachen (Dorn et al., 1968; Dobson et al., 2002; Merlo et al., 2008; Vascellari et al., 2009; Sleeckx et al., 2011). Italienische und britische Studien berichten von einer Inzidenz von ca. 200/100.000 Hunde/Jahr (Dobson et al., 2002; Merlo et al., 2008; Vascellari et al., 2009). Eine signifikante Reduktion der Inzidenz wird in Ländern erreicht, in denen die Ovari(o)-/ Hysterektomie bei jungen Hündinnen regelmäßig eingesetzt wird (Sleeckx et al., 2011). In einer eigenen 3-jährigen klinischen Studie haben wir eine jährliche Inzidenz von ca. 1\% bei insgesamt 9265 Hunden feststellen können. Die meisten caninen Mammatumoren sind epitheliale Tumore, während ein kleinerer Anteil epithelialer und myoepithelialer Herkunft ist, sog. komplexe Karzinome. Ein geringer Anteil der Tumore ist mesenchymaler Herkunft, die aber auch epitheliale Gewebe enthalten können (Karzinosarkom) (Sleeckx et al., 2011).

Humane und canine Mammatumoren weisen viele Ähnlichkeiten, wie Therapiemöglichkeiten, Inzidenz unterschiedlicher Tumorarten sowie persönliche und umweltbedingte Risikofaktoren, auf (Paoloni and Khanna, 2008; Rowell et al., 2011). Darüber hinaus zeigt das humane Genom umfangreichere Homologien mit dem caninen als dem murinen Genom auf (Lindblad-Toh et al., 2005). Aus diesem Grund wird der Hund auch als das geeignetere biomedizinische Model zur Erforschung humaner Tumoren sowie deren Therapie angesehen (Lindblad-Toh et al., 2005; Khanna et al., 2006). Weitere Ähnlichkeiten betreffen die spontane Tumorgenese, genetische Prädisposition, juvenile Adipositas sowie die Altersabhängigkeit (Sleeckx et al., 2011). Bei humanen Mammatumoren wird routinemäßig die Expression der Östrogen/Progesteron-Rezeptoren (ER/PR), des epidermalen Wachstumsfaktor Rezeptors 2 (HER2/ERBB2) sowie basale und myoepitheliale Marker bestimmt (Queiroga et al., 2011). Danach werden die malignen Tumoren klassifiziert in ER/PR positive Typen Luminal A (ERBB2 negativ) und Luminal B (ERBB2 positiv) sowie die ER/PR negativen basalen, ERBB2 positiven und normalen Tumoren. Die gleichen Subtypen wurden auch beim Hund nachgewiesen, wenngleich diese Faktoren nicht routinemäßig klinisch diagnostiziert werden (Gama et al., 2008; Sassi et al., 2010). Die Expression von ER/PR und HER2 in humanen Mammatumoren sind wesentliche Indikatoren für die Prognostik und Tumortherapie (Tsuda, 2009). ER und PR positive Tumoren findet man beim Hund seltener, was gegebenenfalls auf eine geringere hormonelle Abhängigkeit caniner Tumoren schließen lässt (Rutteman et al., 1988b). Eine verminderte ER-Expression wurde in größeren Tumoren mit Lymphknotenbetei- 
ligung und infauster Prognose beschrieben (Nieto et al., 2000). Das beim Menschen üblicherweise als Anti-Östrogen-Medikament eingesetzte Tamoxifen zeigt beim Hund keine Wirkung und auch die Reaktion auf Chemotherapeutika, wie z. B. Doxorubicin und Docetaxel, ist beim Hund unterschiedlich (Morris et al., 1993; Simon et al., 2006; Sleeckx et al., 2011). Aufgrund des sonst eindeutigen prognostischen Werts von ER/PR und HER2/ERB2 werden beim Hund vor allem auch die Tumorgröße, histologische Differenzierung, Lymphknotenbeteiligung und das invasive Wachstum des Tumors als wichtige prognostische Indikatoren verwendet (Sleeckx et al., 2011). Ein direkter Vergleich humaner und caniner Tumorarten ist jedoch wegen des unterschiedlichen Klassifizierungssystems schwierig (Tsuda, 2009). Dennoch entspricht das beim Hund am häufigsten auftretende einfache tubulopapilläre Karzinom am ehesten dem humanen tubulopapillären Karzinomtyp des invasiven ductalen Karzinoms (Hellmen, 2005; Tsuda, 2009). Strandberg und Goodman postulieren, dass die caninen infiltrativen malignen epithelialen Neoplasien der Milchkanäle histologisch dem humanen ductalen Karzinom sehr ähnlich sind (Strandberg and Goodman, 1974). Sarkome oder Karzinosarkome wie beim Hund sind dagegen beim Menschen sehr selten (Hellmen, 2005). Auch gemischte Tumoren mit myoepithelialen Anteilen findet man beim Menschen selten.

Ein Tumorkennzeichen ist $\mathrm{u}$.a. das Auftreten struktureller genomischer Aberrationen mit Veränderungen der Kopienzahlen, sog. copy-number Imbalanzen (CNI), oder Kopienzahl neutraler chromosomaler Umlagerungen (Negrini et al., 2010; Tang et al., 2010; Angstadt et al., 2011; Maeda et al., 2012). Bei humanen Tumoren sind außerdem tumorspezifische chromosomale Veränderungen beschrieben worden (Beroukhim et al., 2010). Da cytogenetische Untersuchungen beim Hund durch die Vielzahl von Chromosomen erschwert sind, eignen sich hier vor allem Verfahren der vergleichenden genomischen Hybridisierung mittels Microarrays (aCGH) (Thomas et al., 2007; Muller et al., 2012). Auf diese Weise konnten erst kürzlich umfangreiche zwischen Hund und Mensch überlappende CNI-Regionen beim Darmkrebs identifiziert werden (Tang et al., 2010). Chromosomale Instabilitäten werden in zunehmendem Umfang beim Menschen durch den Einsatz von Hochdurchsatzsequenzierungen (,next generation sequencing“, NGS) untersucht (Schweiger et al., 2011). Durch die Analyse sog. „paired-end mapping“(PEM) Signaturen von Tumor-DNAs können nicht nur alle Aberration einwandfrei identifiziert sondern auch die genaue chromosomale Lokalisation bestimmt werden.

Zell-freie Nukleinsäuren (cfNA) im Blut wurden erstmals 1948 von Mandel und Métais beschrieben (Mandel and Métais, 1948). Jedoch erst seit dem Nachweis von KRAS Punktmutationen im Blut von Krebspatienten 1994 wurde begonnen cfNAs intensiver vor allem in der Krebsdiagnostik zu untersuchen und anzuwenden (Sorenson et al., 1994; Vasioukhin et al., 1994; Schwarzenbach et al., 2011). Seither wurden nahezu alle tumor-assoziierten genomischen, genetischen und epigenetischen Veränderungen in cfNA untersucht (Schwarzenbach et al., 2011). Hierzu gehören u.a. Mikrosatellitenveränderungen, Punktmutationen, Veränderungen repetitiver Ele- 
mente und DNA-Methylierung (Beck et al., 2010; Leary et al., 2010; McBride et al., 2010). Bemerkenswert ist dabei, dass die Untersuchung von cfNAs im Vergleich zu anderen Verfahren eine einfache, minimal-invasive Diagnostik darstellt. Wir haben in der vorliegenden Studie fünf Hündinnen mit unterschiedlichen Tumoren des Gesäuges mittels NGS untersucht und konnten dabei tumor-spezifische Veränderungen nachweisen, die auch in den cfNAs detektierbar waren (Shaw et al., 2012; Chan et al., 2013).

\section{Material und Methoden}

\section{Klinische Proben}

Tumorgewebe von fünf Hündinnen wurde im Rahmen der tierärztlichen Behandlung in der Poliklinik des Tierärztlichen Instituts der Georg-August Universität Göttingen operativ entfernt. Das Gewebe wurde für die Klassifizierung histopathologisch untersucht. Weitere 15 Hündinnen wurde im Laufe der Studie mittels ddPCR untersucht. Tabelle 1 gibt die Details der untersuchten Proben, histopathologischen Klassifikation sowie das Kastrationsalter der Hündinnen wieder.

Tab. 1. Untersuchte Hündinnen und Tumorklassifikation.

\begin{tabular}{llll}
\hline Tumor-Nr. & Hunderasse & Kastrationsalter & Tumor \\
\hline 30 & Jack Russel Terrier & 13 & Osteosarkom \\
35 & Border Collie & 12 & einfach tubulopapillär \\
40 & Golden Retriever & 10 & einfach tubulopapillär \\
47 & Franz. Bulldogge & 6 & einfach tubulopapillär \\
49 & Weißer Schweizer Schäferhund & 9 & komplexes Karzinom \\
52 & Deutscher Schäferhund & 9 & einfach tubulopapillär \\
60 & Mongrel & 11 & komplexes Karzinom \\
65 & Deutsch. Spaniel & 9 & komplexes Karzinom \\
69 & Mongrel & 14 & einfach solid \\
73 & Golden Retriever & 5 & einfach solid \\
78 & Mongrel & 11 & einfach tubulopapillär \\
81 & Deutsche Bracke & 13 & einfach solid \\
83 & Mongrel & 15 & einfach tubulopapillär \\
86 & Deutsch Drahthaar & 7 & einfach solid \\
92 & Golden Retriever & 10 & Karzinosarkom \\
97 & Labrador Retriever & 7 & einfach tubulopapillär \\
98 & Mongrel & 7 & einfach tubulopapillär \\
99 & Deutscher Schäferhund & 5 & komplexes Karzinom \\
104 & Gebirgsschweißhund & 15 & einfach tubulopapillär \\
108 & Pudelpointer & 8 & einfaches Karzinom \\
\hline
\end{tabular}




\section{Blutentnahme und Extraktion zellfreier und genomischer DNA}

5-6 mL Blut wurden entnommen und bei $4000 \times \mathrm{g}$ für $15 \mathrm{~min}$ und $4{ }^{\circ} \mathrm{C}$ zentrifugiert. Der Überstand wurde in eines neues Reaktionsgefäß überführt und bis zur weiteren Bearbeitung bei $-20^{\circ} \mathrm{C}$ gelagert. Zur Extraktion der genomischen DNA wurden außerdem die Blutzellen (PBMC) bei $-20^{\circ} \mathrm{C}$ aufbewahrt. Nach der operativen Entfernung wurden Teile der Tumoren homogenisiert.

Für die Isolierung der cfDNA wurden die zellulären Restbestandteile einer 0,7 mL Plasmaprobe durch Zentrifugation bei $4000 \mathrm{x}$ g für 20 min entfernt und der Überstand vorsichtig abgenommen. Die cfDNA wurde aus jeweils 0,6 mL gereinigtem Plasma mit dem High Pure Viral Nucleic Acid Extraction Kit (Roche Applied Sciences) gemäß den Angaben des Herstellers isoliert.

Genomische DNA wurde aus $25 \mathrm{mg}$ homogenisiertem Tumorgewebe mit dem QIAGEN Blood and Tissue Kit (Qiagen) gemäß den Angaben des Herstellers extrahiert. Aus den PBMC wurde die DNA nach einem modifizierten Protokoll von Miller isoliert (Miller et al., 1988).

\section{Herstellung der DNA-Bibliotheken und DNA-Sequenzierung}

„Mate-pair“ Bibliotheken wurden für das SOLiD4+ System (Lifetechnologies) wie beschrieben hergestellt (McKernan et al., 2009). Dazu wurde die genomische DNA auf eine Größe von ca. 3 kb fragmentiert und nach der Zirkularisierung, Nick-translation und Adaptorligation als Matrize für eine Emulsions-PCR verwendet. Die Sequenzierung von 40 bp (read 1) und 50 bp (read 2) wurden auf einem SOLiD4+ Objektträger durchgeführt. „Paired-end“ Bibliotheken wurden von 2 Tumoren, 2 entsprechenden cfDNAs und fünf PBMC-Kontrollgenomen angefertigt und sequenziert (50 bp read 1, $35 \mathrm{bp}$ read 2).

Die erhaltenen DNA-Sequenzen wurden gegen das canine Referenzgenom (canFam 2) mit der Bioscope Software (Lifetechnologies) verglichen. Doppelte und qualitativ unzureichende Daten wurden mit PICARD Software eliminiert (http://picard. sourceforge.net).

\section{Vergleichende Untersuchung der DOC („depth of coverage“)}

Zur Bestimmung der Verhältnisse und p-Werte für jedes Tumor/PBMC-Paar wurde das Programm CNV-seq mit normalisierten Lesedichten in den entsprechenden Auswertungsbereichen verwendet (Xie and Tammi, 2009). Die Konfidenzniveaus zur Berechnung der kleinsten Auswertungsbereiche für jede Probe wurden auf $p=0,0001$ und einem Verhältnis von $\log 2=0,6\left(\mathrm{r}^{2}\right)$ festgesetzt. Die erhaltenen Verhältnisse wurden nach Parzen-Rosenblatt geglättet (Rosenblatt, 1956; Parzen, 1962). 


\section{Kartierung von „paired-end“ Signaturen (PEM)}

Aberrante „paired-end“ Signaturen wurden mit dem Programm SVDetect analysiert (Zeitouni et al., 2010). Eine PEM-Signatur wurde als aberrant angesehen, wenn die Enden nicht korrekt im Abstand, der Anordnung oder Orientierung waren. Der Grenzwert für Deletionen und Duplikationen wurde auf 4 Standardabweichungen der mittleren Insertlänge der Bibliothek im Vergleich zum caninen Referenzgenom festgesetzt. Es wurden mindestens 3 informative Sequenzen bei Tumoren und 1 Sequenz bei PBMC als Voraussetzung für die Annahme eines korrekten Ergebnisses ausgewertet. Alle Daten wurden mittels unterschiedlicher Filter bereinigt.

\section{Digitale PCR zur Untersuchung der proximalen CFA27-Deletion}

Zur Analyse der proximalen Deletion von CFA27 in den Tumoren wurde eine „droplet“ digitale PCR (ddPCR) verwendet. $1 \mu \mathrm{g}$ genomische Tumor- und entsprechende PBMC-DNA wurden mit $5 \mathrm{U}$ ApoI in $25 \mu \mathrm{L}$ Volumen gespalten. Die ddPCR wurde mit einem QX100 Droplet Digital PCR System (Bio-Rad) durchgeführt. Die $20 \mu \mathrm{L}$ Reaktionen enthielten $2 \times$ ddPCR Master Mix (Bio-Rad), $900 \mathrm{nmol} / \mathrm{L}$ der entsprechenden PCR-Primer, $250 \mathrm{nmol} / \mathrm{L}$ der Hydrolyseprobe und $60 \mathrm{ng}$ DNA. Die Primer lagen ca. 1700 bp außerhalb des PFDN5-Gens (CFA27.F: CAGGTGCAGCCCCAATAAGA; CFA27.R: CCCCGCTTCTGTACTACGTC; CFA27-Probe: 6-FAM-TTGAGTCGGGGAGCCTGGCG-BHQ1). Als Kontrolle wurde ein Bereich auf CFA32 amplifiziert (CF32.F: AAAAGCCTCCAATCC CCGAG; CFA23.R: CCTGACAGAAAAAGCAGCCC; CFA32-Probe: HEX-CTCCGTGACAAGTC AAGCTCAATAGCCT-BHQ1). Jede $20 \mu \mathrm{L}$ PCR wurde mittels des QX100 Droplet Generators (Bio-Rad) in 20.000 Tropfen einer Wasser-in Öl-Emulsion überführt. Die Reaktionsbedingungen waren $95^{\circ} \mathrm{C}$ für 10 min gefolgt von 50 Zyklen bei jeweils $95^{\circ} \mathrm{C}$ für $30 \mathrm{sec}$ und $62^{\circ} \mathrm{C}$ für $1 \mathrm{~min}$. Nach der PCR wurden die Reaktionen in einen QX100 Droplet-Reader verbracht. Die durchschnittliche Anzahl an ausgewerteten „droplets“ betrug 14.121 (SD: 995). Die Kopienzahl der entsprechenden Fragmente wurde mit der Software Quantasoft ${ }^{\mathrm{TM}}$ bestimmt (Pinheiro et al., 2012).

\section{Quantifizierung individueller Umlagerungen}

Weitere digitale PCR wurden zur Untersuchung tumor-spezifischer Umlagerungen in der Tumor-DNA und cfDNA entwickelt. Zur Kontrolle wurde ein Fragment aus dem FGF5-Gen auf CFA32 verwendet (FGF5.F: GAGAGGTAGTGAGAAGGTCAAAG; FGF5.R: ACAATTCACATTATGGATGCCAAG). Die Reaktionen wurden in $5 \mu \mathrm{L}$ Volumen mit 0,25 U Faststart DNA Polymerase (Roche), $200 \mu \mathrm{mol} / \mathrm{L}$ dNTPs, 500 nmol/L der entsprechenden Primer durchgeführt. DNA wurde in einer Konzentration von 0,5 Genomäquivalenten beigemischt (1,3 ng insges.). Die Reaktionen wurden bei $95^{\circ} \mathrm{C}$ für $10 \mathrm{~min}$ gestartet und dann mit 50 Zyklen bei $95{ }^{\circ} \mathrm{C}$ für $30 \mathrm{sec}, 62^{\circ} \mathrm{C}$ für $30 \mathrm{sec}$ und $72{ }^{\circ} \mathrm{C}$ für $30 \mathrm{sec}$ auf einem Light Cycler 480 (Roche) fortgeführt. Die Detektion erfolgte durch Zugabe von EvaGreen in einer 384-Platte, die zu einer Hälfte die Umlagerungs-spezifischen Primer 
und zur anderen Hälfte die FGF5-Kontroll-PCR-Primer enthielt. Die Kopienzahl jeder Probe wurde auf Grundlage einer Poisson-Verteilung berechnet. Das Verhältnis der Bruchpunkt-Fragmente und der FGF5-Fragmente wurde für jede Tumor- und cfDNA bestimmt.

\section{Ergebnisse}

\section{Sequenzabdeckung und Mutationsanalyse}

Die „mate-pair“ Sequenzierung der DNA von 5 Primärtumoren und entsprechenden PBMC-Proben ergaben im Durchschnitt insgesamt 37 Millionen auswertbare Läufe. Die niedrigste Anzahl von Läufen wurde bei der Tumor- und PBMC-DNA von Tier Nr. 52 erhalten (28,4 M; 22,9 M) und die höchste Anzahl bei Tumor 49 (52,9 M). Zusätzliche „paired-end“ Sequenzierungen ergaben jeweils 88 M, 87 M, 65 M, und 98 M Läufe für T49, T47, PBMC49 und PBMC47. Von den cfDNA wurden bei Tier Nr. 49 und Tier Nr. 47 jeweils $31 \mathrm{M}$ und $15 \mathrm{M}$ Läufe erhalten. Tabelle 2 faßt die Ergebnisse der Sequenzierungen zusammen.

Tab. 2. Zusammenfassung der Sequenzierungsergebnisse.

\begin{tabular}{lccc}
\hline Probe & Sequenzierung & Läufe & mittlere Insert-Größe (SD) \\
\hline Tumor 49 & mate-pair & 52958301 & $2554(719)$ \\
PBMC 49 & mate-pair & 46573332 & $2331(638)$ \\
Tumor 47 & mate-pair & 37059440 & $2415(744)$ \\
PBMC 47 & mate-pair & 42991422 & $2381(620)$ \\
Tumor 30 & mate-pair & 28996426 & $2013(748)$ \\
PBMC 30 & mate-pair & 35798374 & $2349(612)$ \\
Tumor 52 & mate-pair & 28435620 & $2593(735)$ \\
PBMC 52 & mate-pair & 22915754 & $2279(679)$ \\
Tumor 35 & mate-pair & 29418741 & $2269(780)$ \\
PBMC 35 & mate-pair & 47255421 & $2487(655)$ \\
Tumor 49 & paired-end & 88240507 & $88(55)$ \\
PBMC 49 & paired-end & 64811016 & $90(49)$ \\
cfDNA 49 & paired-end & 30960138 & $145(67)$ \\
Tumor 47 & paired-end & 86628229 & $128(66)$ \\
PBMC 47 & paired-end & 98267227 & $136(85)$ \\
cfDNA 47 & paired-end & 14857474 & $129(21)$ \\
PBMC 40 & paired-end & 98964511 & $144(99)$ \\
PBMC 52 & paired-end & 65341166 & $89(42)$ \\
PBMC 27 & paired-end & 86829099 & $133(65)$ \\
\hline
\end{tabular}




\section{Untersuchung der Abdeckungstiefe („depth of coverage“, DOC)}

Mit dem Programm CNV-seq wurden die Kopienzahländerungen in den fünf Tumoren ermittelt und im Verhältnis zur nukleären DNA der PBMC wiedergegeben. Dabei wurden jeweils Bereiche von 37.969 bp bei Tier Nr. 49 und 75.380 bp bei Tier Nr. 52 verwendet. Zwei Regionen mit einer Kopienzahlerhöhung konnten für T49 bei Chromosom CFA27 und CFA35 nachgewiesen werden. Beide Chromosomen zeigten ein komplexes Muster von umfangreichen Amplifikationen und Deletionen. Darüber hinaus konnte eine Deletion der Region 42,6-47,9 Mbp auf CFA11 nachgewiesen werden, die das Tumor-Suppressor-Cluster $C D K N 2 A-C D K N 2 B$ enthält. Der gleiche Bereich war auch bei T47 deletiert.

Während T49 ein sehr heterogenes Muster an Veränderungen aufwies, zeigte T47 eher umfangreiche Hypoploidien mit vollständigem Verlust der Chromosomen CFA2, 3, 5, 11, 18, 19, 21, 27, 29, 30, 33, 37 und 38 sowie der Verdopplung von CFA1.

Die höchsten Kopienzahländerungen wurden bei T30 detektiert. T30 zeigte außerdem einige Ähnlichkeiten mit T47, da diesem Tumor auch die Chromosomen 27, 29, 37 und 38 fehlten. T30 wies darüber hinaus umfangreiche Deletionen in CFA3 auf. Dagegen war CFA13, welches die Oncogene $c M Y C$ und KIT trägt, amplifiziert. Bei den untersuchten Tumoren zeigte nur T30 eine Duplikation der Gene HER2/ERBB2. In T52 konnten keine umfangreicheren Veränderungen festgestellt werden und T35 erschien bis auf vier kleinere Deletionen, die aber vermutlich auf Sequenzierungsartefakte zurückzuführen waren, unverändert. Die drei Tumoren des einfachen tubulopapillären Typs (T47, T52, T35) zeigten keine substantiellen Ähnlichkeiten, abgesehen von einer geringfügig höheren Anzahl an Deletionen in T47 und T52.

\section{Nachweis struktureller Aberrationen}

Strukturelle Aberrationen wurden mit dem Programm SVDetect analysiert und durch unterschiedliche Filter geprüft (Zeitouni et al., 2010). Tabelle 3 gibt die validierten Aberrationen der fünf Tumoren wieder. Insgesamt wurden 54 somatische Umlagerungen identifiziert.

Tab. 3. Strukturelle Veränderungen in fünf caninen Mammatumoren.

\begin{tabular}{lrrrrr}
\hline Tumor & T49 & T47 & T30 & T52 & T35 \\
\hline Insgesamt & 23 & 1 & 28 & 2 & 0 \\
Duplikation & 2 & 0 & 3 & 0 & 0 \\
Insertion & 0 & 0 & 0 & 1 & 0 \\
Deletion & 5 & 0 & 20 & 0 & 0 \\
Translokation & 9 & 1 & 0 & 1 & 0 \\
Inversion & 7 & 0 & 5 & 0 & 0 \\
\hline
\end{tabular}


Ähnlich wie bei den Kopienzahländerungen zeigten T49 und T30 die meisten strukturellen Veränderungen. In T47 konnte nur eine Translokation nachgewiesen werden. In T52 konnten eine Translokation und eine Insertionen detektiert werden, während T35 keine Veränderungen aufwies. Verglichen zu Untersuchungen beim Menschen, wiesen die caninen Tumoren weniger strukturelle Veränderungen auf. Die Anzahl strukturellen Veränderungen in den Tumoren ist sowohl beim Mensch als auch Hund sehr heterogen und es gibt durchaus Tumoren die keine Veränderungen zeigen. Abbildung 1 zeigt einen Circos Plot von T49 in dem die verschiedenen Aberrationen zu sehen sind. Deutlich zu erkennen ist eine Anhäufung von Bruchpunkten auf CFA27 und CFA35. Mehrere interchromosomale Translokationen haben zwischen diesen beiden Chromosomen stattgefunden. Die Bruchpunktregionen zeigen komplexe Umlagerungen mit vielzähligen Änderungen der Kopienzahl. Durch die komplexen Aberrationen auf CFA27 wurde eine Reihe von Genen unterbrochen, z. B. PPFIBP1 und SLC2A3 durch Translokation und SOX5, ANO2, MANSC1 und CLSTN3 durch Inversion. Durch die Inversion von $S O X 5$ und ANO2 kam es zu einer Fusion dieser beiden Gene in den Introns 4 und 11, wobei jedoch auf Grund der invertierten Orientierung von ANO2 nicht mit einem Fusionsprotein zu rechnen ist. Eine weitere durch komplexe Aberrationen gekennzeichnete Region befindet sich auf CFA11:42.62-47.92 Mbp in der Nähe der Tumor-Suppressor-Region CDKN2A-CDKN2B.

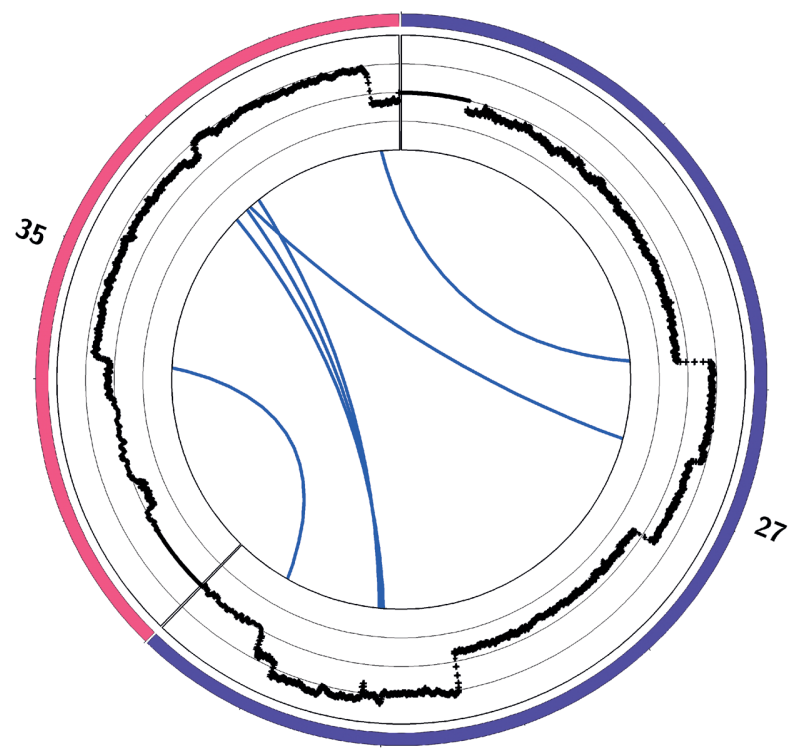

Abb. 1. Strukturelle Aberrationen zwischen CFA27 und CFA35 in Tumor 49. 


\section{Genotypisierung einer Deletion auf CFA27}

Während der größere Anteil an Aberrationen sich in den Tumorgenomen sehr heterogen darstellte, konnte eine Deletion am proximalen Ende von CFA27 bei vier der Tumoren identifiziert werden. Diese Region konnte auf ca. 8,8 Mbp eingegrenzt werden. Neben $\mathrm{u}$. a. einigen olfaktorischen und Keratin-Genen befinden sich in dieser Region die beiden Tumor-assoziierten Gene PFDN5 und TMBIM6. Der BAX Inhibitor TMBIM6 fungiert als Oncoprotein, in dem er den apoptotischen Zelltod verhindert (Grzmil et al., 2006). PFDN5 wurde als Tumor-Supressor beschrieben und stellt damit ein sehr gutes funktionelles Kandidatengen für die Tumorgenese dar (Fujioka et al., 2001).

Mit einer digitalen PCR wurden die Daten der DOC überprüft und dazu 15 weitere Tumorgenome auf die CFA27-Deletion untersucht. Die CFA27-Deletion konnte in den bereits analysierten 4 Tumoren und außerdem in 6 der 15 zusätzlichen Tumoren bestätigt werden. Somit konnte bei 50\% der untersuchten Tumoren die CFA27Deletion detektiert werden, die aber sonst mit keinem anderen Parameter korrelierte (z. B. Tumorklassifikation, Tumorstadium, ER Status, HER2 Genamplifikation). Lediglich der Ki-67 Index war signifikant höher bei Tumoren mit der CFA27-Deletion.

\section{Nachweis der Tumorsignaturen in zellfreier plasmatischer DNA}

Für jedes der vier sequenzierten Tumorgenome wurden spezifische PCR-Tests zum Nachweis der chromosomalen Bruchpunkte entwickelt. Bei allen Hunden konnten die tumor-spezifischen Bruchpunkte in der zellfreien plasmatischen DNA vor der chirurgischen Entfernung des Tumors nachgewiesen werden. Von Tier Nr. 49 wurden außerdem 83 und 89 Wochen nach der Operation Blutproben entnommen und cfDNA isoliert. Dabei konnte 83 Wochen $p$. op. noch 5\% und 89 Wochen $p$. op. noch 4,2\% tumor-spezifische Bruchpunkte-Amplicons im Verhältnis zu den Kontrollen nachgewiesen werden. Durch eine computertomographische Untersuchung konnten gezeigt werden, dass Tier Nr 49. eine Lungenmetastase ausgebildet hatte, die die Persistenz der tumor-spezifischen Amplicons erklärte (Abb. 2).

\section{Diskussion}

Die „mate-pair“ Sequenzierung von fünf unterschiedlichen caninen Mammatumoren ergab ein sehr heterogenes Muster genomischer Aberrationen. Selbst bei den drei tubulopapillären Tumoren konnten keine einheitlichen Veränderungen nachgewiesen werden. Genomische Instabilität und Mutationen gehören zu den Charakteristika der Tumorgenese, wobei die chromosomalen Instabilitäten (CNI) zu den häufigsten Veränderungen zählen und in vollständige oder segmentale Aneuploidien unterteilt werden können (Geigl et al., 2008; Hanahan and Weinberg, 2011; Martin et al., 2012). Während vollständige Aneuploidien durch eine inkorrekte Segregation bei der Mitose entstehen, bilden sich segmentale Aneuploidien, die auch als strukturelle Verände- 


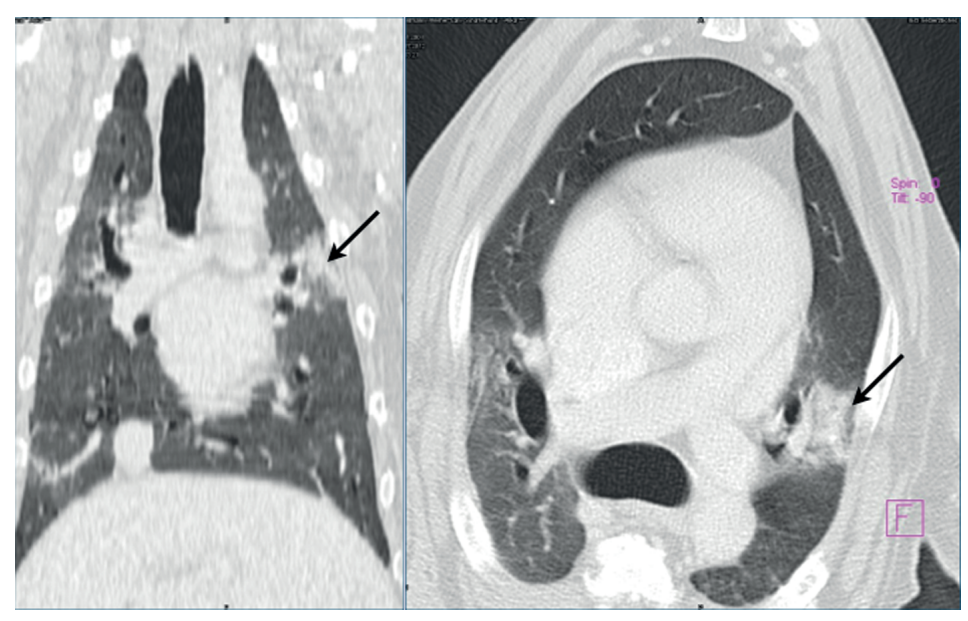

Abb. 2. Computertomographische Untersuchung der Hündin 49. Die Abbildung zeigt zwei Schnittebenen des thorakalen Bereichs der Hündin 49. Die Pfeile zeigen die Lage der Metastase im Lungenparenchym 89 Wochen $p$. op.

rungen/Aberrationen bezeichnet werden, durch Doppelstrangbrüche während der Replikation aus (Geigl et al., 2008). Zwei der hier untersuchten Tumorgenome (T49, T30) zeigten eine Vielzahl struktureller Veränderungen auf subchromosomaler Ebene, während bei T47 chromosomale Instabilität beobachtet wurden. Hypoploidie, wie in diesem Tumor, findet man im Gegensatz zum Menschen bei caninen Mammatumoren relativ häufig. Dagegen zeigen humane Mammakarzinome sehr oft Amplifikationen der Chromosomen HSA1q, HSA11q, HSA8q und HSA16p (Hellmen et al., 1988; Rutteman et al., 1988a; Hicks et al., 2006; Kwei et al., 2010). Im Genom des Tumors T49 konnten komplexe interchromosomale Umlagerungen innerhalb einiger Chromosomen nachgewiesen werden, welche auf eine sog. Chromothripsis hinweisen. Dieses erst kürzlich in humanen Tumoren entdeckte Phänomen beschreibt einen initialen zellulären Vorgang, bei dem es zu einer Anhäufung chromosomaler Brüche während der Mitose kommt (Stephens et al., 2011; Liu et al., 2012; Maher and Wilson, 2012). Die hier beteiligten caninen Chromosomen CFA27 und CFA35 sind mit den humanen Chromosomen HSA12 und HSA6 syntänisch. Während bei HSA6 häufiger komplexe Umlagerungen in Mammatumoren beschrieben wurden, scheint HSA12 dafür weniger bekannt zu sein (Hicks et al., 2006). Die einzige auf CFA27 gelegene Fusion der Gene SOX5 und ANO2 in T49 wurde in humanen Tumoren bisher nicht beschrieben und scheint daher funktionell eher keine Bedeutung zu haben. Diese Fusion ist vermutlich das Ergebnis aus den sonstigen umfangreichen Umlagerungen innerhalb des Genoms und repräsentiert eine individuelle Aberration dieses Tumors.

Die meisten Umlagerungen und Aneuploidien konnten in T30 nachgewiesen werden. Das dort nachweisbare Muster von Aberrationen deutet auf einen sog. Mutatorphänotyp hin, bei dem es durch die Mutation vieler an der chromosomalen Stabilität 
beteiligten Gene zu einer Kaskade weiterer Aberrationen kommt (Loeb et al., 2003; Liu et al., 2012). Eine andere Interpretation wäre, dass durch die Aneuploidien und dem damit verbundenen Verlust einer großen Vielzahl auch an der Zellteilung und Chromosomenstabilität beteiligter Gene, die weitreichenden strukturellen Veränderungen ausgelöst werden (Fabarius et al., 2003). Welcher der beiden Vorgänge nun tatsächlich ursächlich für die Tumorgenese ist bleibt unklar, aber es ist sicherlich nicht falsch davon auszugehen, dass beide Prozesse zusammenwirken (Boerkamp et al., 2012).

Die beiden Tumoren T52 und T35 wiesen die geringsten Aberrationen auf. Auch beim Menschen wurden Mammatumore beschrieben, die nur geringe Ausmaße von Umlagerungen und Veränderungen zeigen. Solche „flachen“ Profile könnten aber auch durch einen höheren Anteil noch nicht neoplastischer Zellen im Bioptat bedingt sein (Hicks et al., 2006).

Insgesamt zeigten die in den caninen Mammatumoren nachgewiesenen Veränderungen große Ähnlichkeiten mit denen beim Menschen. Die Veränderungen in T47 entsprechen dem einfachen Typ, während T30 dem komplexen Typ I oder „sawtooth“ Typ und T49 dem komplexen Typ II oder „firestorm“ Typ entsprechen (Hicks et al., 2006). Daneben gibt es aber auch spezies-spezifische Aberrationen, die auf evolutionär instabile hypervariable Regionen zurückzuführen sind, so wie sie z. B. beim Mensch für Chromosom 8p23.1 gezeigt werden konnten (Tang et al., 2010).

Bei der Suche nach durch Deletionen und Amplifikationen betroffener kausaler Tumor- oder Tumorsuppressorgene fiel besonders die proximale Region von CFA27 auf, die bei ca. 50\% der Tumorgenome deletiert war. In dieser Region befindet sich das Tumorsuppressorgen PFDN5, welches ein Modulator von cMYC ist (Hagio et al., 2006). Eine reduzierte Expression von PFDN5 wurde $\mathrm{u}$. a. bei durch Progestine induzierten caninen Mammahyperplasien nachgewiesen (Rao et al., 2009). Die PFDN5 Deletion war nicht mit erhöhter ER Aktivität oder HER2 Amplifikation assoziiert, korrelierte aber mit dem KI-67 Index.

Zur Etablierung eines einfachen nicht invasiven Nachweisverfahrens wurde neben den Tumorgenomen auch die zellfreie plasmatische DNA (cfDNA) untersucht. Für die tumor-spezifischen Bruchpunkte wurden PCR-Tests entwickelt und damit für den Hund erstmalig eine Quantifizierung der Bruchpunkte im Plasma durchgeführt. Aus Untersuchungen beim Mensch ist bekannt, dass die Amplifikation von Bruchpunkten in cfDNA eine hervorragende Methode zur Kontrolle des postoperativen Verlaufs darstellt (Leary et al., 2010). Bei unseren Untersuchungen fiel bei einer Hündin die Persistenz der tumor-spezifischen Bruchpunktamplicons auch noch 89 Wochen nach der Operation auf. Bei einer computertomographischen Nachuntersuchung der Hündin konnte eine Metastase in der Lunge nachgewiesen werden. Damit konnte zum ersten Mal beim Hund die Einsatzmöglichkeit der cfDNA in der Tumordiagnostik und postoperativen Verlaufskontrolle gezeigt werden.

Die zur Zeit noch arbeitsintensive und kostspielige Identifikation individueller chromosomaler Bruchpunkte und der damit verbundenen initialen Komplettsequenzierung von Tumorgenomen ist gegenwärtig noch nachteilig für einen routinemäßigen 
Einsatz dieser Verfahren in der Veterinärmedizin. Darüber hinaus werden bei Hündinnen mit Mammatumoren bisher üblicherweise keine Verlaufskontrollen durchgeführt. Dennoch weisen unsere Ergebnisse daraufhin, dass auch in der Veterinärmedizin zukünftig der Einsatz der zellfreien plasmatischen DNA als Biomarker in der Diagnostik mit Sicherheit an Bedeutung gewinnen wird.

\section{Danksagung}

Wir danken Stefan Balzer, Sarah Bierau, Melanie Scharfenstein für die ausgezeichnete technische Unterstützung und Cord Bielke für seinen Beitrag bei der Datenauswertung.

\section{Literatur}

Angstadt, A.Y., Motsinger-Reif, A., Thomas, R., Kisseberth, W.C., Guillermo Couto, C., Duval, D.L., Nielsen, D.M., Modiano, J.F. and Breen, M., 2011. Characterization of canine osteosarcoma by array comparative genomic hybridization and RT-qPCR: signatures of genomic imbalance in canine osteosarcoma parallel the human counterpart. Genes Chromosomes Cancer 50, 859-74.

Beck, J., Urnovitz, H.B., Mitchell, W.M. and Schutz, E., 2010. Next generation sequencing of serum circulating nucleic acids from patients with invasive ductal breast cancer reveals differences to healthy and nonmalignant controls. Mol Cancer Res 8, 335-42.

Beroukhim, R., Mermel, C.H., Porter, D., Wei, G., Raychaudhuri, S., Donovan, J., Barretina, J., Boehm, J.S., Dobson, J., Urashima, M., Mc Henry, K.T., Pinchback, R.M., Ligon, A.H., Cho, Y.J., Haery, L., Greulich, H., Reich, M., Winckler, W., Lawrence, M.S., Weir, B.A., Tanaka, K.E., Chiang, D.Y., Bass, A.J., Loo, A., Hoffman, C., Prensner, J., Liefeld, T., Gao, Q., Yecies, D., Signoretti, S., Maher, E., Kaye, F.J., Sasaki, H., Tepper, J.E., Fletcher, J.A., Tabernero, J., Baselga, J., Tsao, M.S., Demichelis, F., Rubin, M.A., Janne, P.A., Daly, M.J., Nucera, C., Levine, R.L., Ebert, B.L., Gabriel, S., Rustgi, A.K., Antonescu, C.R., Ladanyi, M., Letai, A., Garraway, L.A., Loda, M., Beer, D.G., True, L.D., Okamoto, A., Pomeroy, S.L., Singer, S., Golub, T.R., Lander, E.S., Getz, G., Sellers, W.R. and Meyerson, M., 2010. The landscape of somatic copy-number alteration across human cancers. Nature 463, 899-905.

Boerkamp, K.M., Rutteman, G.R., Kik, M.J., Kirpensteijn, J., Schulze, C. and Grinwis, G.C., 2012. Nuclear DNA-Content in Mesenchymal Lesions in Dogs: Its Value as Marker of Malignancy and Extent of Genomic Instability. Cancers (Basel) 4, 1300-17.

Chan, K.C., Jiang, P., Zheng, Y.W., Liao, G.J., Sun, H., Wong, J., Siu, S.S., Chan, W.C., Chan, S.L., Chan, A.T., Lai, P.B., Chiu, R.W. and Lo, Y.M., 2013. Cancer genome scanning in plasma: detection of tumor-associated copy number aberrations, single-nucleotide variants, and tumoral heterogeneity by massively parallel sequencing. Clin Chem 59, 211-24.

Dobson, J.M., Samuel, S., Milstein, H., Rogers, K. and Wood, J.L., 2002. Canine neoplasia in the UK: estimates of incidence rates from a population of insured dogs. J Small Anim Pract 43, 240-6.

Dorn, C.R., Taylor, D.O., Schneider, R., Hibbard, H.H. and Klauber, M.R., 1968. Survey of animal neoplasms in Alameda and Contra Costa Counties, California. II. Cancer morbidity in dogs and cats from Alameda County. J Natl Cancer Inst 40, 307-18.

Fabarius, A., Hehlmann, R. and Duesberg, P.H., 2003. Instability of chromosome structure in cancer cells increases exponentially with degrees of aneuploidy. Cancer Genet Cytogenet 143, 59-72. 
Fujioka, Y., Taira, T., Maeda, Y., Tanaka, S., Nishihara, H., Iguchi-Ariga, S.M., Nagashima, K. and Ariga, H., 2001. MM-1, a c-Myc-binding protein, is a candidate for a tumor suppressor in leukemia/lymphoma and tongue cancer. J Biol Chem 276, 45137-44.

Gama, A., Alves, A. and Schmitt, F., 2008. Identification of molecular phenotypes in canine mammary carcinomas with clinical implications: application of the human classification. Virchows Arch 453, 123-32.

Geigl, J.B., Obenauf, A.C., Schwarzbraun, T. and Speicher, M.R., 2008. Defining 'chromosomal instability'. Trends Genet 24, 64-9.

Grzmil, M., Kaulfuss, S., Thelen, P., Hemmerlein, B., Schweyer, S., Obenauer, S., Kang, T.W. and Burfeind, P., 2006. Expression and functional analysis of Bax inhibitor-1 in human breast cancer cells. J Pathol 208, 340-9.

Hagio, Y., Kimura, Y., Taira, T., Fujioka, Y., Iguchi-Ariga, S.M. and Ariga, H., 2006. Distinct localizations and repression activities of MM-1 isoforms toward c-Myc. J Cell Biochem 97, 145-55.

Hanahan, D. and Weinberg, R.A., 2011. Hallmarks of cancer: the next generation. Cell 144, 646-74.

Hellmen, E., 2005. Complex mammary tumours in the female dog: a review. J Dairy Res $72 \mathrm{Spec}$ No, 90-7.

Hellmen, E., Lindgren, A., Linell, F., Matsson, P. and Nilsson, A., 1988. Comparison of histology and clinical variables to DNA ploidy in canine mammary tumors. Vet Pathol 25, 219-26.

Hicks, J., Krasnitz, A., Lakshmi, B., Navin, N.E., Riggs, M., Leibu, E., Esposito, D., Alexander, J., Troge, J., Grubor, V., Yoon, S., Wigler, M., Ye, K., Borresen-Dale, A.L., Naume, B., Schlicting, E., Norton, L., Hagerstrom, T., Skoog, L., Auer, G., Maner, S., Lundin, P. and Zetterberg, A., 2006. Novel patterns of genome rearrangement and their association with survival in breast cancer. Genome Res 16, 1465-79.

Khanna, C., Lindblad-Toh, K., Vail, D., London, C., Bergman, P., Barber, L., Breen, M., Kitchell, B., McNeil, E., Modiano, J.F., Niemi, S., Comstock, K.E., Ostrander, E., Westmoreland, S. and Withrow, S., 2006. The dog as a cancer model. Nat Biotechnol 24, 1065-6.

Kwei, K.A., Kung, Y., Salari, K., Holcomb, I.N. and Pollack, J.R., 2010. Genomic instability in breast cancer: pathogenesis and clinical implications. Mol Oncol 4, 255-66.

Leary, R.J., Kinde, I., Diehl, F., Schmidt, K., Clouser, C., Duncan, C., Antipova, A., Lee, C., McKernan, K., De La Vega, F.M., Kinzler, K.W., Vogelstein, B., Diaz, L.A., Jr. and Velculescu, V.E., 2010. Development of personalized tumor biomarkers using massively parallel sequencing. Sci Transl Med 2, 20 ra14.

Lindblad-Toh, K., Wade, C.M., Mikkelsen, T.S., Karlsson, E.K., Jaffe, D.B., Kamal, M., Clamp, M., Chang, J.L., Kulbokas, E.J., 3rd, Zody, M.C., Mauceli, E., Xie, X., Breen, M., Wayne, R.K., Ostrander, E.A., Ponting, C.P., Galibert, F., Smith, D.R., DeJong, P.J., Kirkness, E., Alvarez, P., Biagi, T., Brockman, W., Butler, J., Chin, C.W., Cook, A., Cuff, J., Daly, M.J., DeCaprio, D., Gnerre, ., Grabherr, M., Kellis, M., Kleber, M., Bardeleben, C., Goodstadt, L., Heger, A., Hitte, C., Kim, L., Koepfli, K.P., Parker, H.G., Pollinger, J.P., Searle, S.M., Sutter, N.B., Thomas, R., Webber, C., Baldwin, J., Abebe, A., Abouelleil, A., Aftuck, L., Ait-Zahra, M., Aldredge, T., Allen, N., An, P., Anderson, S., Antoine, C., Arachchi, H., Aslam, A., Ayotte, L., Bachantsang, P., Barry, A., Bayul, T., Benamara, M., Berlin, A., Bessette, D., Blitshteyn, B., Bloom, T., Blye, J., Boguslavskiy, L., Bonnet, C., Boukhgalter, B., Brown, A., Cahill, P., Calixte, N., Camarata, J., Cheshatsang, Y., Chu, J., Citroen, M., Collymore, A., Cooke, P., Dawoe, T., Daza, R., Decktor, K., DeGray, S., Dhargay, N., Dooley, K., Dooley, K., Dorje, P., Dorjee, K., Dorris, L., Duffey, N., Dupes, A., Egbiremolen, O., Elong, R., Falk, J., Farina, A., Faro, S., Ferguson, D., Ferreira, P., Fisher, S., FitzGerald, M., et al., 2005. Genome sequence, comparative analysis and haplotype structure of the domestic dog. Nature 438, 803-19. 
Liu, P., Carvalho, C.M., Hastings, P.J. and Lupski, J.R., 2012. Mechanisms for recurrent and complex human genomic rearrangements. Curr Opin Genet Dev 22, 211-20.

Loeb, L.A., Loeb, K.R. and Anderson, J.P., 2003. Multiple mutations and cancer. Proc Natl Acad Sci USA 100, 776-81.

Maeda, J., Yurkon, C.R., Fujisawa, H., Kaneko, M., Genet, S.C., Roybal, E.J., Rota, G.W., Saffer, E.R., Rose, B.J., Hanneman, W.H., Thamm, D.H. and Kato, T.A., 2012. Genomic instability and telomere fusion of canine osteosarcoma cells. PLoS One 7, e43355.

Maher, C.A. and Wilson, R.K., 2012. Chromothripsis and human disease: piecing together the shattering process. Cell 148, 29-32.

Mandel, P. and Métais, P., 1948. Les acides nucleiques du plasma sanguin chez l’homme. C R Seances Soc Biol Fil 142, 241-3.

Martin, J.W., Squire, J.A. and Zielenska, M., 2012. The genetics of osteosarcoma. Sarcoma 2012, 627254.

McBride, D.J., Orpana, A.K., Sotiriou, C., Joensuu, H., Stephens, P.J., Mudie, L.J., Hamalainen, E., Stebbings, L.A., Andersson, L.C., Flanagan, A.M., Durbecq, V., Ignatiadis, M., Kallioniemi, O., Heckman, C.A., Alitalo, K., Edgren, H., Futreal, P.A., Stratton, M.R. and Campbell, P.J., 2010. Use of cancer-specific genomic rearrangements to quantify disease burden in plasma from patients with solid tumors. Genes Chromosomes Cancer 49, 1062-9.

McKernan, K.J., Peckham, H.E., Costa, G.L., McLaughlin, S.F., Fu, Y., Tsung, E.F., Clouser, C.R., Duncan, C., Ichikawa, J.K., Lee, C.C., Zhang, Z., Ranade, S.S., Dimalanta, E.T., Hyland, F.C., Sokolsky, T.D., Zhang, L., Sheridan, A., Fu, H., Hendrickson, C.L., Li, B., Kotler, L., Stuart, J.R., Malek, J.A., Manning, J.M., Antipova, A.A., Perez, D.S., Moore, M.P., Hayashibara, K.C., Lyons, M.R., Beaudoin, R.E., Coleman, B.E., Laptewicz, M.W., Sannicandro, A.E., Rhodes, M.D., Gottimukkala, R.K., Yang, S., Bafna, V., Bashir, A., MacBride, A., Alkan, C., Kidd, J.M., Eichler, E.E., Reese, M.G., De La Vega, F.M. and Blanchard, A.P., 2009. Sequence and structural variation in a human genome uncovered by short-read, massively parallel ligation sequencing using two-base encoding. Genome Res 19, 1527-41.

Merlo, D.F., Rossi, L., Pellegrino, C., Ceppi, M., Cardellino, U., Capurro, C., Ratto, A., Sambucco, P.L., Sestito, V., Tanara, G. and Bocchini, V., 2008. Cancer incidence in pet dogs: findings of the Animal Tumor Registry of Genoa, Italy. J Vet Intern Med 22, 976-84.

Miller, S.A., Dykes, D.D. and Polesky, H.F., 1988. A simple salting out procedure for extracting DNA from human nucleated cells. Nucleic Acids Res 16, 1215.

Morris, J.S., Dobson, J.M. and Bostock, D.E., 1993. Use of tamoxifen in the control of canine mammary neoplasia. Vet Rec 133, 539-42.

Muller, M.H., Reimann-Berg, N., Bullerdiek, J. and Murua Escobar, H., 2012. Genetic characterization of dogs via chromosomal analysis and array-based comparative genomic hybridization (aCGH). Tierarztl Prax Ausg K Kleintiere Heimtiere 40, 55-8.

Negrini, S., Gorgoulis, V.G. and Halazonetis, T.D., 2010. Genomic instability-an evolving hallmark of cancer. Nat Rev Mol Cell Biol 11, 220-8.

Nieto, A., Pena, L., Perez-Alenza, M.D., Sanchez, M.A., Flores, J.M. and Castano, M., 2000. Immunohistologic detection of estrogen receptor alpha in canine mammary tumors: clinical and pathologic associations and prognostic significance. Vet Pathol 37, 239-47.

Paoloni, M. and Khanna, C., 2008. Translation of new cancer treatments from pet dogs to humans. Nat Rev Cancer 8, 147-56.

Parzen, E., 1962. On the estimation of a probability density and the mode. The Annals of Mathematical Statistics 33, 1065-1076.

Pinheiro, L.B., Coleman, V.A., Hindson, C.M., Herrmann, J., Hindson, B.J., Bhat, S. and Emslie, K.R., 2012. Evaluation of a droplet digital polymerase chain reaction format for DNA copy number quantification. Anal Chem 84, 1003-11. 
Queiroga, F.L., Raposo, T., Carvalho, M.I., Prada, J. and Pires, I., 2011. Canine mammary tumours as a model to study human breast cancer: most recent findings. In Vivo 25, 455-65.

Rao, N.A., van Wolferen, M.E., Gracanin, A., Bhatti, S.F., Krol, M., Holstege, F.C. and Mol, J.A., 2009. Gene expression profiles of progestin-induced canine mammary hyperplasia and spontaneous mammary tumors. J Physiol Pharmacol 60 Suppl 1, 73-84.

Rosenblatt, M., 1956. Remarks on some nonparametric estimates of a density function. The Annals of Mathematical Statistics 37, 832-837.

Rowell, J.L., McCarthy, D.O. and Alvarez, C.E., 2011. Dog models of naturally occurring cancer. Trends Mol Med 17, 380-8.

Rutteman, G.R., Cornelisse, C.J., Dijkshoorn, N.J., Poortman, J. and Misdorp, W., 1988a. Flow cytometric analysis of DNA ploidy in canine mammary tumors. Cancer Res 48, 3411-7.

Rutteman, G.R., Misdorp, W., Blankenstein, M.A. and van den Brom, W.E., 1988b. Oestrogen (ER) and progestin receptors $(P R)$ in mammary tissue of the female dog: different receptor profile in non-malignant and malignant states. Br J Cancer 58, 594-9.

Sassi, F., Benazzi, C., Castellani, G. and Sarli, G., 2010. Molecular-based tumour subtypes of canine mammary carcinomas assessed by immunohistochemistry. BMC Vet Res 6, 5.

Schwarzenbach, H., Hoon, D.S. and Pantel, K., 2011. Cell-free nucleic acids as biomarkers in cancer patients. Nat Rev Cancer 11, 426-37.

Schweiger, M.R., Kerick, M., Timmermann, B. and Isau, M., 2011. The power of NGS technologies to delineate the genome organization in cancer: from mutations to structural variations and epigenetic alterations. Cancer Metastasis Rev 30, 199-210. Shaw, J.A., Page, K., Blighe, K., Hava, N., Guttery, D., Ward, B., Brown, J., Ruangpratheep, C., Stebbing, J., Payne, R., Palmieri, C., Cleator, S., Walker, R.A. and Coombes, R.C., 2012. Genomic analysis of circulating cell-free DNA infers breast cancer dormancy. Genome Res 22, 220-31.

Simon, D., Schoenrock, D., Baumgartner, W. and Nolte, I., 2006. Postoperative adjuvant treatment of invasive malignant mammary gland tumors in dogs with doxorubicin and docetaxel. J Vet Intern Med 20, 1184-90.

Sleeckx, N., de Rooster, H., Veldhuis Kroeze, E.J., Van Ginneken, C. and Van Brantegem, L., 2011. Canine mammary tumours, an overview. Reprod Domest Anim 46, 1112-31.

Sorenson, G.D., Pribish, D.M., Valone, F.H., Memoli, V.A., Bzik, D.J. and Yao, S.L., 1994. Soluble normal and mutated DNA sequences from single-copy genes in human blood. Cancer Epidemiol Biomarkers Prev 3, 67-71.

Stephens, P.J., Greenman, C.D., Fu, B., Yang, F., Bignell, G.R., Mudie, L.J., Pleasance, E.D., Lau, K.W., Beare, D., Stebbings, L.A., McLaren, S., Lin, M.L., McBride, D.J., Varela, I., Nik-Zainal, S., Leroy, C., Jia, M., Menzies, A., Butler, A.P., Teague, J.W., Quail, M.A., Burton, J., Swerdlow, H., Carter, N.P., Morsberger, L.A., lacobuzio-Donahue, C., Follows, G.A., Green, A.R., Flanagan, A.M., Stratton, M.R., Futreal, P.A. and Campbell, P.J., 2011. Massive genomic rearrangement acquired in a single catastrophic event during cancer development. Cell 144, 27-40.

Strandberg, J.D. and Goodman, D.G., 1974. Animal model of human disease: canine mammary neoplasia. Am J Pathol 75, 225-8.

Tang, J., Le, S., Sun, L., Yan, X., Zhang, M., Macleod, J., Leroy, B., Northrup, N., Ellis, A., Yeatman, T.J., Liang, Y., Zwick, M.E. and Zhao, S., 2010. Copy number abnormalities in sporadic canine colorectal cancers. Genome Res 20, 341-50.

Thomas, R., Duke, S.E., Bloom, S.K., Breen, T.E., Young, A.C., Feiste, E., Seiser, E.L., Tsai, P.C., Langford, C.F., Ellis, P., Karlsson, E.K., Lindblad-Toh, K. and Breen, M., 2007. A cytogenetically characterized, genome-anchored 10-Mb BAC set and CGH array for the domestic dog. J Hered 98, 474-84. 
Tsuda, H., 2009. Gene and chromosomal alterations in sporadic breast cancer: correlation with histopathological features and implications for genesis and progression. Breast Cancer 16, 186-201.

Vascellari, M., Baioni, E., Ru, G., Carminato, A. and Mutinelli, F., 2009. Animal tumour registry of two provinces in northern Italy: incidence of spontaneous tumours in dogs and cats. BMC Vet Res 5 , 39.

Vasioukhin, V., Anker, P., Maurice, P., Lyautey, J., Lederrey, C. and Stroun, M., 1994. Point mutations of the $\mathrm{N}$-ras gene in the blood plasma DNA of patients with myelodysplastic syndrome or acute myelogenous leukaemia. Br J Haematol 86, 774-9.

Xie, C. and Tammi, M.T., 2009. CNV-seq, a new method to detect copy number variation using high-throughput sequencing. BMC Bioinformatics 10, 80.

Zeitouni, B., Boeva, V., Janoueix-Lerosey, I., Loeillet, S., Legoix-ne, P., Nicolas, A., Delattre, O. and Barillot, E., 2010. SVDetect: a tool to identify genomic structural variations from paired-end and mate-pair sequencing data. Bioinformatics 26, 1895-6. 